\title{
Extreme Sensitivity to Detuning for Globally Coupled Phase Oscillators
}

\author{
Peter Ashwin, ${ }^{1}$ Oleksandr Burylko, ${ }^{2}$ Yuri Maistrenko, ${ }^{2,3}$ and Oleksandr Popovych ${ }^{3}$ \\ ${ }^{1}$ Department of Mathematical Sciences, University of Exeter, Exeter EX4 4QE, United Kingdom \\ ${ }^{2}$ Institute of Mathematics, National Academy of Sciences of Ukraine, 01601 Kyiv, Ukraine \\ ${ }^{3}$ Institute of Medicine and Virtual Institute of Neuromodulation, Research Centre Jülich, 52425 Jülich, Germany
}

(Received 4 August 2005; published 6 February 2006)

\begin{abstract}
We discuss the sensitivity of a population of coupled oscillators to differences in their natural frequencies, i.e., to detuning. We argue that for three or more oscillators, one can get great sensitivity even if the coupling is strong. For $N$ globally coupled phase oscillators we find there can be bifurcation to extreme sensitivity, where frequency locking can be destroyed by arbitrarily small detuning. This extreme sensitivity is absent for $N=2$, appears at isolated parameter values for $N=3$ and $N=4$, and can appear robustly for open sets of parameter values for $N \geq 5$ oscillators.
\end{abstract}

DOI: 10.1103/PhysRevLett.96.054102

During the last two decades, the study of networks of coupled oscillators has become a very popular and promising area of modern nonlinear science. Growing interest has been stimulated by a wide variety of applications such as Josephson junction arrays [1], semiconductor laser arrays [2], coupled chemical reactions [3], and cardiac pacemaker cells [4]. A crucial feature of such systems is their ability to model transition between synchronized and desynchronized states. This transition can be identified, e.g., as a loss of frequency locking between oscillators in the network. In neurophysiology, the appearance of phase synchronization of oscillatory neurons, in particular, brain areas, is associated with diseases such as Parkinson's disease or epilepsy [5]. Uncovering the mechanisms of the pathological neuronal synchrony represents a challenging task in the development of novel deep brain stimulation techniques for therapy of such diseases [6].

Work starting with Kuramoto [7,8] (see the review [9]) has examined generic properties of the interplay between coupling strength and frequency spread for coupled phase oscillators,

$$
\dot{\theta}_{i}=\omega_{i}+\frac{K}{N} \sum_{j=1}^{N} g\left(\theta_{i}-\theta_{j}\right)
$$

where $\left(\theta_{1}, \ldots, \theta_{N}\right) \in T^{N}$ and $T^{1}=[0,2 \pi)$. We examine this equation for the coupling function $g$ of [10]

$$
g(\phi):=-\sin (\phi+\alpha)+r \sin (2 \phi) .
$$

Many studies have considered the case $r=0$ and $\alpha=0$ in the thermodynamic limit $N \rightarrow \infty$, and with frequencies $\omega_{i}$ chosen independently from a fixed distribution $[8,9]$. Some details of the very rich dynamics that can appear in a system of a few coupled phase oscillators at a loss of stability of a synchronized attractor have recently been examined in [11]. Chaotic dynamics of the phases has been found and studied in the Kuramoto model for weak and intermediate coupling strength [12], while [13] investigates the dynamics of "repulsive interaction."
PACS numbers: 05.45.Xt

For the standard Kuramoto model [8] with $r=\alpha=0$, reduction of the coupling strength $K$ will result in greater sensitivity of synchronized behavior to variation in the natural frequencies $\omega_{i}$; this will manifest as frequency mismatch in the response if the $\omega_{i}$ are sufficiently different. For (1) with coupling function (2), the situation is different and much richer: Extremely small detuning may desynchronize phase dynamics even without decoupling. Indeed, by varying $\alpha$ and $r$ one can get an exchange of stability of in-phase and antiphase states. We find that the transition between the two phase locked states is typically accompanied by so-called extreme sensitivity to detuning. It means that near the transition, phase locking can be destroyed by introducing arbitrary small differences of the natural frequencies of individual oscillators. The extreme sensitivity phenomenon is found for system (1) beginning from dimension $N=3$, and we believe it is a robust phenomenon as it is driven by the existence of robust heteroclinic attractors. We present detailed mechanisms of the phenomenon and its relation to slow switching oscillations.

It is known that (1) displays a variety of attractors and bifurcations, including cluster states, periodic orbits, tori, and heteroclinic cycles that may be robust [10]. Other coupling functions of the form $g\left(\theta_{i}, \theta_{j}\right)$ can permit, for example, the possibility of oscillation death [14], but system (1) is justifiable for weak to moderate values of coupling [15]. The dynamics of this system was investigated in Refs. [10,16] for identical oscillators, $\omega_{i}=\omega$. For $N \geq 4$ oscillators there can be robust slow oscillations between cluster states, caused by the presence of attracting heteroclinic cycles. We show how these heteroclinic cycles can be used to explain the emergence of extreme sensitivity to detuning in system (1).

To quantify the sensitivity to detuning we define a vector $\delta=\left(\Delta_{1}, \ldots, \Delta_{N-1}\right)$ by $\Delta_{i}=\omega_{i+1}-\omega_{1}$. Fixing all parameters in system (1) except $\delta$, we define sensitivity to detuning to be the supremum of $\Omega$ such that $\|\delta\|<\Omega$ implies all attractors for the system of oscillators have phase differences that remain bounded. If $\Omega$ is small, the 
system has a lower threshold of sensitivity to detuning. If $\Omega=0$, we say the system shows extreme sensitivity to detuning.

We find that loss of stability of in-phase oscillation $\theta_{1}=$ $\cdots=\theta_{N}$ for identical oscillators (1) is associated with a minimum of the sensitivity $\Omega$ on fixing $r, K$, and varying $\alpha$. The nature of this minimum depends critically on the number of oscillators. For $N=2$ we find that the minimum is positive while for $N \geq 3$ the minimum is zero (extreme sensitivity) and remains exponentially close to zero for parameter values near loss of stability of in-phase oscillation. For $N \geq 5, \Omega$ can become zero for a whole interval of parameter values, indicating robust extreme sensitivity.

We say two oscillators $\theta_{i}$ and $\theta_{j}$ have a bounded phase difference if $\left|\theta_{j}(t)-\theta_{i}(t)\right|$ is bounded uniformly for $t>0$. If $\omega_{i}=\omega$ (so $\Delta_{i} \equiv 0$ ) then the sets $S_{i j}$ defined by those phases where $\theta_{i}=\theta_{j}$ are invariant for all $i \neq j$ and form codimension one barriers to the dynamics. Hence, in the absence of detuning, we can consider the flow in the socalled canonical invariant region [15] defined by $\theta_{1} \leq$ $\theta_{2} \leq \theta_{3} \leq \cdots \leq \theta_{N} \leq \theta_{1}+2 \pi$. There must be a bounded phase difference in this case regardless of the detailed dynamics, and regardless of $N$. This argument does not hold as soon as the $\omega_{i}$ are different. The model (1) with $r=\alpha=0$ and $\Delta_{i}=0$ has a degenerate bifurcation on changing $K$ at $K=0$. For $K>0$ the in-phase oscillation is stable and antiphase states (i.e., where the order parameter $R=N^{-1}\left\|\sum_{k=1}^{N} \exp \left(i \theta_{k}\right)\right\|$ satisfies $R=$ 0 ) are unstable. For $K<0$ the only attractors are the antiphase states, and these are degenerate manifolds of neutrally stable periodic solutions [13].
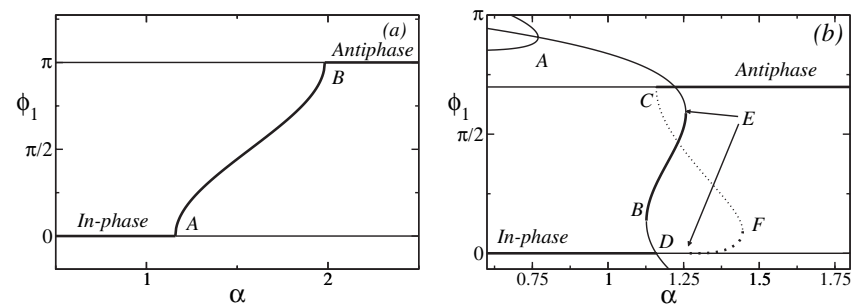

FIG. 1. Schematic bifurcation diagrams for (1) with $r=0.2$, $K=1$ and (a) $N=2$, (b) $N=3$. For $N=2$ in (a), obtained analytically, there are pitchfork bifurcations at $A$ and $B$ located at $\alpha=\arccos ( \pm 2 r)$ and branches of attracting symmetry-broken equilibria connecting $A$ and $B$. For $N=3$ in (b), the antiphase state (where the order parameter $R=0$ ) bifurcates at $C$ to give a branch of unstable periodic orbits (thin dotted curve). This has a saddle node at $F$ with a stable periodic orbit (bold dotted curve), terminating at a global saddle-node heteroclinic bifurcation at $E$. For limit cycles the minimum value over the cycle is plotted. Lines show equilibria; bold indicates that they are attracting. The bifurcations are $A(\alpha=0.7691)$, pitchfork; $B(\alpha=1.1269)$, saddle node of equilibria; $C(\alpha=1.1593)$, Hopf from antiphase state; $D(\alpha=1.1593), S_{3}$-transcritical bifurcation; $E(\alpha=$ 1.2574), global saddle-node heteroclinic; $F(\alpha=1.4452)$, saddle node of limit cycles. Branch $A E B D$ lies within $\phi_{2}=0$; there are symmetrically related branches in $\phi_{1}=0$ and $\phi_{1}=\phi_{2}$.
We now consider the more general case with $r \neq 0$ and $\alpha \neq 0$ but still identical oscillators, first for the cases $N=$ 2 and 3. The complexity of bifurcation increases dramatically with $N$ and so for $N=4,5$ we focus only on robust heteroclinic attractors that can appear in these cases. By scaling time we set $K=1$.

For $N=2$ and $\phi:=\theta_{2}-\theta_{1}$, Eq. (1) reduces to

$$
\dot{\phi}=\Delta_{1}-\sin \phi \cos \alpha+r \sin 2 \phi,
$$

from which one can determine that for fixed $\Delta_{1}=0$ and $0<r<0.5$ there are solutions $\phi=0$ (in-phase) and $\phi=\pi$ (antiphase) for all $\alpha$. There are also branches of solutions with $\phi= \pm \arccos \left(\frac{\cos \alpha}{2 r}\right)$ that appear at $\alpha=$ $\arccos 2 r$ and disappear at $\alpha=\arccos (-2 r)$ via supercritical pitchfork bifurcations. These transfer stability from $\phi=0$ to $\phi=\pi$ via the branches of symmetrybroken solutions as illustrated in Fig. 1(a). At $r=0$ the branches are vertical and hence degenerate. If $N=2$ and $0<r<0.5$, then for any $\alpha$ one can find the sensitivity $\Omega=\min \left\{\left\|F_{+}\right\|,\left\|F_{-}\right\|\right\}$, where $F_{+}\left(F_{-}\right)$is the global maximum (minimum) of $F(\phi)=-\sin \phi \cos \alpha+$ $r \sin 2 \phi$. One can observe that these extrema occur at a value of $\pm \arccos \left(\frac{\cos \alpha \pm \sqrt{\cos ^{2} \alpha+32 r^{2}}}{8 r}\right)$. The sensitivity $\Omega$ as a function of $\alpha$ is shown in Fig. 4 below for $r=0.2$, including the case $N=2$. One can see that (i) this has minima at $\alpha=\pi / 2$ (where $\left|F_{+}\right|=\left|F_{-}\right|$) corresponding to the $\sin \phi$ term vanishing in Eq. (3) and (ii) the minimum value is given by $r$ and so is nonzero.

For $N=3$ we can reduce system (1) to a twodimensional flow on a torus, given by an ODE for the phase differences $\phi_{1}=\theta_{2}-\theta_{1}$ and $\phi_{2}=\theta_{3}-\theta_{1}$. Fixing $r=0.2$ and $\Delta_{1}=\Delta_{2}=0$, the bifurcation diagram on varying $\alpha$ is a lot more complicated than that for $N=2$. The bifurcations for $N=3$ as found by numerical path following with XPPAUT [17] are listed $A-F$ in Fig. 1(b). The bifurcation at $E$ at $\alpha=\alpha_{0}=1.2574, r=0.2$ is of particular interest as it gives extreme sensitivity to detuning (qualitatively similar bifurcations occur for $0.156<$ $r<0.375)$.

Figure 2 plots the phase differences for $N=3$ using $\xi=$ $\theta_{1}+e^{2 \pi i / 3} \theta_{2}+e^{4 \pi i / 3} \theta_{3}$ (permutations of the oscillators correspond to rotations and reflections in the phase space that preserve an equilateral triangle [18]). The lift of this torus of phase differences to $\mathbb{R}^{2}$ can be represented by reflecting triangles from Fig. 2 along their edges to build a triangulation of the plane. The sides of the triangles represent the invariant subspaces $\phi_{1}=0, \phi_{2}=0$, and $\phi_{1}=\phi_{2}$ bounding the canonical invariant region for $\Delta_{i}=$ 0 . There is a stable node $P$ and saddles $Q$ and $R$ on the invariant subspaces [Fig. 2(a)]. At $\alpha=\alpha_{0}$, a saddle-node bifurcation of $P$ and $R$ occurs [Fig. 2(b)], and at $\alpha>\alpha_{0}$ there is a stable limit cycle $L$ created near the union of unstable manifolds of the remaining saddles $Q$ [Fig. 2(c)].

At the bifurcation [Fig. 2(b)] we assume the eigenvalues at $Q$ are $\lambda_{1}<0$ and $\lambda_{2}>0$ while at $P$ they are $\lambda_{3}<0$ and 0 . We derive an approximating map $F: \Pi_{1} \rightarrow \Pi_{1}^{\prime}$ with 

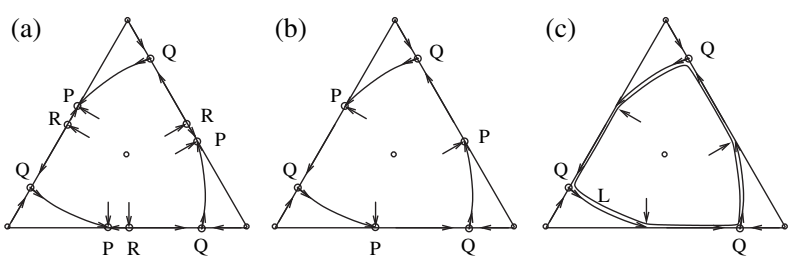

FIG. 2. Flow near the global saddle-node heteroclinic bifurcation for $N=3$ with $r=0.2$ shown schematically in the plane $\xi=\theta_{1}+e^{2 \pi i / 3} \theta_{2}+e^{4 \pi i / 3} \theta_{3}$. The phase portraits show (a) before the bifurcation for $\alpha<\alpha_{0}=1.2574$, (b) at the bifurcation for $\alpha=\alpha_{0}$, and (c) after the bifurcation for $\alpha>$ $\alpha_{0}$ there is an attracting limit cycle $L$. This limit cycle is very close to the invariant subspaces, meaning that small detuning can give an attractor with unbounded phase differences.

$F(a)=c$, for $0<a \ll 1$, where the symbols are as shown in Fig. 3(a). Since $\Pi_{1}$ and $\Pi_{1}^{\prime}$ are symmetrically related, the return map to $\Pi_{1}$ is given simply by $F^{3}$. Consider a parameter $\nu$ that unfolds the saddle node at $P$, such that there are two hyperbolic fixed points near $P$ for $\nu<0$ and none for $\nu>0$. Clearly for $\nu \leq 0$ trajectories starting close to the invariant subspaces, i.e., having $a$ small enough, will not intersect $\Pi_{1}^{\prime}$ as they will be trapped at $P$. For $\nu>0$ suppose a trajectory starting on $\Pi_{1}$ at distance $a$ from the stable manifold of $Q$ returns to $\Pi_{1}$ at a distance $\tilde{a}$ from the stable manifold. Approximation by a linear flow near $Q$ gives to leading order $\tilde{a}=K_{1} \exp \left[-K_{2} \nu^{-1 / 2}\right]$ for positive constants $K_{1}$ and $K_{2}$. This implies there is a superstable periodic orbit created close to the invariant subspace containing $Q$. In terms of the original variables $\nu=\left|\alpha-\alpha_{0}\right|$ we have $\tilde{a}=K_{1} \exp \left[-K_{2}\left|\alpha-\alpha_{0}\right|^{-1 / 2}\right]$ independent of $a$ to leading order in $\left|\alpha-\alpha_{0}\right|$. The period $T$ of the cycle
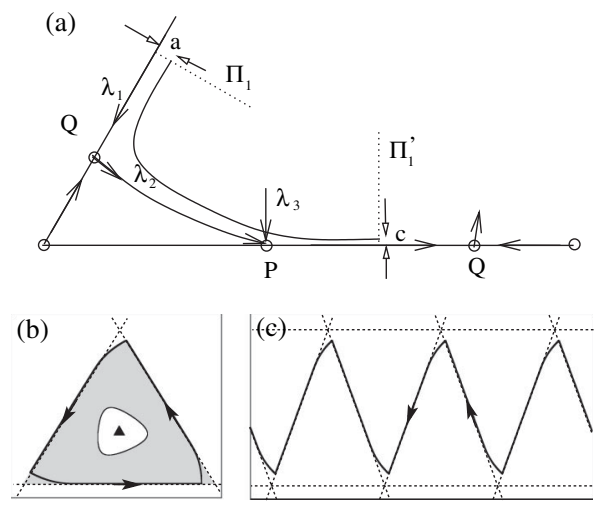

FIG. 3. (a) Construction of the return map from $\Pi_{1}$ to $\Pi_{1}^{\prime}$; this map is defined only when the saddle node $P$ has been destroyed by a small perturbation [case (c) in Fig. 2]. (b) Phase portrait of system (1) with $N=3$ in the complex plane $(\operatorname{Re}(\xi), \operatorname{Im}(\xi))$ for $r=0.2, K=1, \alpha=1.28$, and $\Delta_{1}=\Delta_{2}=\Delta=4.1 \times 10^{-7}$. Bold solid curve depicts a stable limit cycle with the basin of attraction indicated by gray. At the center of the triangle is a coexisting stable antiphase state. (c) For a slightly larger detuning $\Delta=4.2 \times 10^{-7}$ we obtain an attracting periodic orbit that is unbounded in $\xi$. The subspaces $\phi_{1}=0, \phi_{2}=0$, and $\phi_{1}=\phi_{2}$, which are invariant for $\Delta=0$, are depicted by dashed lines. scales as $T=K_{3}\left|\alpha-\alpha_{0}\right|^{-1 / 2}$, implying that the period goes to infinity very slowly as we approach the bifurcation point illustrated in Fig. 2 (as $\alpha \rightarrow \alpha_{0}$ from above). Such a limit cycle is illustrated in Fig. 3(b) (bold curve). At the saddle-node heteroclinic bifurcation the attracting web of interconnecting invariant manifolds winds nontrivially around the torus. These arise on lifting the invariant manifolds shown in Fig. 2(b) (where the sides of the triangles denote the invariant subspaces $\phi_{1}=0, \phi_{2}=0$, and $\phi_{1}=$ $\phi_{2}$, manifolds of $P$ and $Q$ and their symmetric images) to the torus [see Fig. 3(c), dashed lines]. This web of connections cause arbitrarily small detuning $\Delta$ to give attractors near this web that have unbounded phase differences. Such a desynchronized attractor is shown in Fig. 3(c), replacing a bounded stable limit cycle in Fig. 3(b). Moreover, for $\alpha>\alpha_{0}$ the attracting periodic orbits are exponentially close to the invariant manifolds [Figs. 2(c) and 3(b)] meaning that we can estimate $\Omega=$ $O\left(\exp \left[-K\left|\alpha-\alpha_{0}\right|^{-1 / 2}\right]\right)$ for $\alpha>\alpha_{0}$. For $\alpha<\alpha_{0}$ we have $\Omega=O\left(\left|\alpha-\alpha_{0}\right|\right)$ because of the saddle-node pair. This agrees with scaling of sensitivity $\Omega$ near $\alpha=\alpha_{0}$, as illustrated in Fig. 4(a) for the cases $N=3,4,5$ [see also Figs. 4(b) and 4(c) for scaling of $\Omega$ as $\alpha \rightarrow \alpha_{0}$ from above, for $N=3$ and $N=5$, respectively) [19].

For $N=4$ the bifurcation diagram is considerably more complicated, and one can find open sets of parameter values where the only attractor is a robust heteroclinic cycle showing slow oscillations [19] between cluster states of the form $(\theta, \theta, \phi, \phi)$ with $\theta \neq \phi$. Rather than discuss this in detail, we refer the reader to Refs. [10,16]. These attractors consist of saddle periodic orbits and their onedimensional unstable manifolds. Typical initial conditions are attracted to this union of connecting orbits, meaning that they oscillate between symmetrically related saddle periodic orbits while showing "slowing down" of the oscillation period. The calculations for $N=4$ and $r=$ 0.2 in Fig. 4 indicate there is a bifurcation to extreme sensitivity at $\alpha_{1}=1.15928$.

The bifurcation illustrated in Fig. 2 and the transcritical homoclinic bifurcation [18] have extreme sensitivity at the point of bifurcation for the same reason; they give attracting heteroclinic networks that allow winding of trajectories around the torus. The dynamics in [13] shows extreme sensitivity to detuning for larger $N$; however, in this case the exceptional nature of the coupling gives nongeneric behavior, and there are many neutrally stable periodic orbits in the dynamics for $N \geq 4$. In our system, the scenarios giving rise the sensitivity are nondegenerate, except for the assumption of global coupling.

For $N=5$ a new phenomenon appears. Figure 5(b) illustrates a heteroclinic attractor robustly connecting one fundamental region of the torus to the next (this was found in Ref. [20]), which allows unbounded growth of phase differences for arbitrarily small detuning. We contrast this to $N=4$ where the heteroclinic attractor is robust but does not link fundamental regions. Numerical calculations of 

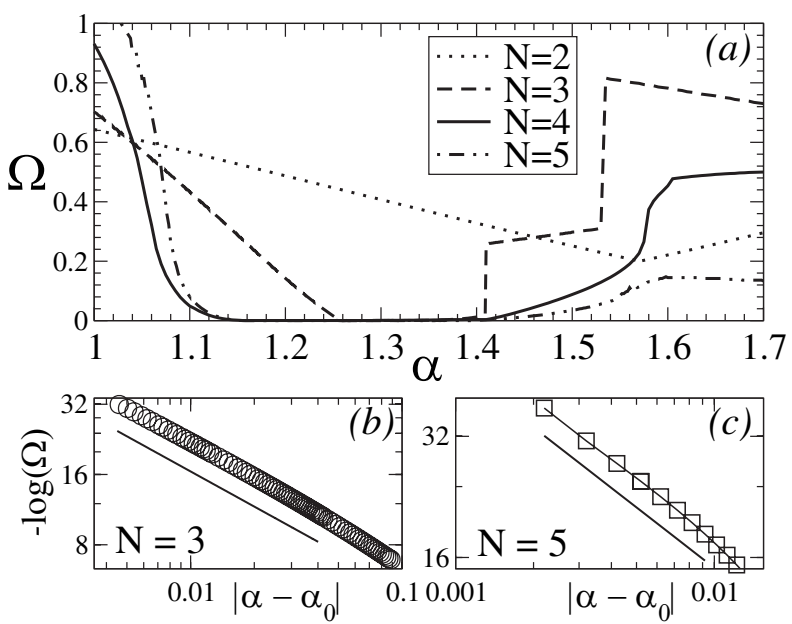

FIG. 4. Diagram showing sensitivity $\Omega$ for $N=2,3,4$, and 5 coupled phase oscillators for $K=1$ and $r=0.2$ and varying $\alpha$. $\Omega$ gives the smallest amplitude detuning $\Delta$ such that at least one attractor is not phase bounded (multistability is common in this region). Observe that $N=2$ remains a bounded distance from $\Delta=0$ while the cases $N=3,4$, and 5 go to zero and remain close to there for a range of $\alpha$ (in fact, for $N=5$ it remains zero to numerical accuracy for a range of $\alpha$ ). The plots (b) and (c) show, for the cases $N=3$ and $N=5$, respectively, the values of $-\log (\Omega)$ plotted versus $\left|\alpha-\alpha_{0}\right|$ in $\log -\log$ scale. The straight lines are obtained by direct fit of the data and have a slope -0.5 , agreeing with the predicted scaling near the bifurcation in Fig. 2(b).

the system with $N=5, K=1$, and $r=0.2$ show there is an interval of values $\alpha \in[1.21,1.41]$ for which the trajectories of the system for small detuning $\Delta$ are spuriously attracted by a saddle fixed point [Fig. 5(a)]. This gives nonzero but incorrect values of the sensitivity $\Omega$ that are reduced by higher precision calculations [Fig. 5(b)]. Hence, for $N=5$ we conclude there are open regions of robust extreme sensitivity. We believe this is typical for $N \geq 5$.
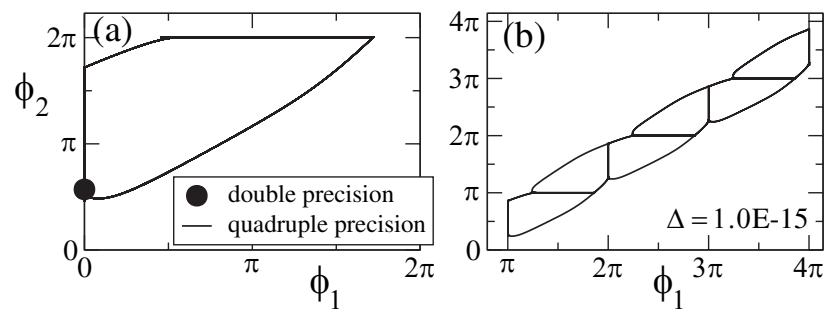

FIG. 5. The system (1) with $N=5, \alpha=1.411, r=0.2$, and $K=1$ at onset of the region of robust extreme sensitivity. (a) Attractors calculated using double and quadruple precision for $\Delta=4.35 \times 10^{-7}$ and $\Delta=9.45 \times 10^{-17}$, respectively. Calculation with quadruple precision gives $\Omega \approx 9.5 \times 10^{-17}$, whereas the double precision gives $\Omega \approx 4.4 \times 10^{-7}$. The double precision calculation shows anomalous attraction to a saddle point. (b) Calculations for a fixed $\Delta=10^{-15}$ using quadruple precision give an attractor that stays close to a robust heteroclinic network with unbounded phase differences.
In conclusion, we show in this Letter that extreme sensitivity to detuning of the natural frequencies is a generic property in coupled oscillator systems, and can occur without decoupling the oscillators. It will appear near exchange stability of the in-phase and antiphase synchronized states, and manifests itself as a violation of frequency synchronization between individual oscillators by arbitrary small detuning. We argue that in networks of $N=5$ oscillators the extreme sensitivity can appear robustly, i.e., for open sets in parameter space.

Coupled oscillators, under certain generic types of coupling, can have attractors that respond very sensitively to differences in the oscillators, whether these are differences in detuning, signal, or noise inputs, and even if the coupling is neither weak nor degenerate. There can be a variety of different bifurcation scenarios leading to extreme sensitivity; these are clearly of great interest.

[1] K. Wiesenfeld, P. Colet, and S. H. Strogatz, Phys. Rev. Lett. 76, 404 (1996); Phys. Rev. E 57, 1563 (1998).

[2] G. Kozyreff, A. G. Vladimirov, and P. Mandel, Phys. Rev. Lett. 85, 3809 (2000).

[3] W. Wang, I. Z. Kiss, and J. L. Hudson, Chaos 10, 248 (2000); Phys. Rev. Lett. 86, 4954 (2001).

[4] C.S. Peskin, Mathematical Aspects of Heart Physiology (Courant Institute, New York, 1975).

[5] A. Nini et al., J. Neurophysiol. 74, 1800 (1995).

[6] P. A. Tass, Phase Resetting in Medicine and Biology (Springer, Berlin, 1999); Europhys. Lett. 53, 15 (2001); Phys. Rev. E 66, 036226 (2002).

[7] Y. Kuramoto, Springer Lecture Notes in Physics (Springer, Berlin, 1975), Vol. 39, pp. 420-422.

[8] Y. Kuramoto, Chemical Oscillations, Waves and Turbulence (Springer-Verlag, Berlin, 1984).

[9] S. H. Strogatz, Physica (Amsterdam) 143D, 1 (2000).

[10] D. Hansel, G. Mato, and C. Meunier, Phys. Rev. E 48, 3470 (1993).

[11] Y. Maistrenko et al., Phys. Rev. Lett. 93, 084102 (2004).

[12] O. V. Popovych, Y.L. Maistrenko, and P. A. Tass, Phys. Rev. E 71, 065201(R) (2005).

[13] L. S. Tsimring et al., Phys. Rev. Lett. 95, 014101 (2005).

[14] S. De Monte, F. d'vidio, and E. Mosekilde, Phys. Rev. Lett. 90, 054102 (2003).

[15] P. Ashwin and J. W. Swift, J. Nonlinear Sci. 2, 69 (1992).

[16] H. Kori and Y. Kuramoto, Phys. Rev. E 63, 046214 (2001); H. Kori, Phys. Rev. E 68, 021919 (2003).

[17] G. B. Ermentrout, A Guide to XPPAUT for Researchers and Students (SIAM, Pittsburgh, 2002).

[18] P. Ashwin, G. P. King, and J. W. Swift, Nonlinearity 3, 585 (1990).

[19] For $N=2$ the sensitivity $\Omega$ was obtained analytically. For $N=3,4,5$ the calculations were performed by starting from values of $\Delta_{i}=\Delta>0$, where a desynchronized attractor exists [as in Fig. 3(c)], and then following this attractor with decreasing $\Delta$ up to the bifurcation moment $\Delta=\Omega$, where all phase differences become bounded.

[20] P. Ashwin and J. Borresen, Phys. Rev. E 70, 026203 (2004). 